\title{
¿Hay dilemas propios de la mujer en psicoanálisis? Lo sabido no pensado ${ }^{1}$
}

\author{
Rosario Castaño Catalá ${ }^{2}$ \\ Instituto de Psicoterapia Relacional, Madrid
}

\begin{abstract}
A través de una revisión de facetas del pensamiento contemporáneo, y del psicoanálisis a lo largo de su evolución, este trabajo se interroga acerca del papel que juega la madre y la mujer en su deseo de encontrar un orden simbólico, una madre que es capaz de poner también orden y una mujer que tiene deseos propios. Se concluye que hay dilemas propios y comunes a todas las mujeres en el psicoanálisis. Cada persona (mujer, hombre, transexual, queer, etcétera) necesita y quiere ser oída y sostenida y este deseo se expresa mediante significantes de género, como un problema de identificaciones femeninas y masculinas en una misma persona.
\end{abstract}

Palabras clave: Mujer, Psicoanálisis, Dilemas.

Through a review of facets of contemporary thought, and of psychoanalysis throughout its evolution, this work asks about the role played by mother and woman in their desire to find a symbolic order, a mother who is capable of also put order and a woman who has her own desires. It is concluded that there are dilemmas of their own and common to all women in psychoanalysis. Each person (woman, man, transsexual, queer, etc.) needs and wants to be heard and sustained and this desire is expressed through gender signifiers, as a problem of female and male identifications in the same person.

Key Words: Women, Psychoanalysis, Dilemmas.

English Title: Are there dilemmas typical of women in psychoanalysis? The known not thought

Cita bibliográfica / Reference citation:

Castaño Catalá, R. (2020). ¿Hay dilemas propios de la mujer en psicoanálisis? Lo sabido no pensado. Clínica e Investigación Relacional, 14 (1): 203-215. [ISSN 1988-2939] [Recuperado de www.ceir.info ]

DOI: $10.21110 / 19882939.2020 .140112$

\footnotetext{
${ }^{1}$ Leída como conferencia en el ciclo "Mujer y Psicoanálisis" en Madrid, 4 de octubre 2019, en el Instituto de Psicoterapia Relacional.

2 Rosario Castaño, psicóloga clínica, psicoterapeuta, sexóloga, presidenta de la Sección de Psicoterapias Psicoanalíticas de Federación de Española de Asociaciones de Psicoterapeutas (FEAP). Vicepresidenta del Instituto de Psicoterapia Relacional (IPR) Práctica privada en el centro médico Instituto Palacios (IP).
} 
Es un honor participar en la inauguración del ciclo de conferencias del Instituto de Psicoterapia Relacional, del curso 2019-20, y lo es por muchos motivos, entre ellos, porque soy una gran defensora de los espacios de formación extras y abiertos que el Instituto pone al servicio de los alumnos y también del público en general, como es el caso de este ciclo de conferencias.

También defiendo lo institucional, los psicoterapeutas sabemos que tenemos una profesión compleja y solitaria, y el hecho de compartir espacios comunes, y de tener un sentido de pertenencia es saludable para desarrollar nuestra actividad, además de una garantía para nuestras carreras profesionales. El Instituto de Psicoterapia Relacional (IPR) es miembro de varias asociaciones nacionales e internacionales, entre ellas, de la Federación Española de Asociaciones de Psicoterapeutas (FEAP) donde se establecen los criterios mínimos para la acreditación de los psicoterapeutas en formación, así como la formación continuada de los psicoterapeutas acreditados y los didactas ${ }^{1}$.

Me interesa el tema de este ciclo "Mujer y psicoanálisis" porque yo trabajo en el ámbito privado en un centro édico dedicado a la salud integral de la mujer ${ }^{2}$, y veo fundamentalmente mujeres de las que he aprendido y sigo aprendiendo cada día, y a pesar de los años sigo teniendo curiosidad por la condición humana en general y por cada paciente en particular; decía Erich Fromm ${ }^{3}$ que la curiosidad es el punto de partida de la sabiduría, yo no sé si conseguiré algún día sabiduría, pero mientras tanto me sigo sorprendiendo con cada paciente, y en cada sesión y no me enfoco tanto en tener respuestas como en encontrar preguntas, y poder, así, crear un espacio donde se puedan formular tanto dudas como dilemas ${ }^{4}$. La psicoterapia es una de las pocas posibilidades institucionales que tenemos para ofrecer un espacio en el que poder preguntarse y hablar de sí mismo/ de sí misma, y del mundo en el que se vive.

Me he propuesto traer una serie de reflexiones que me planteo en el día a día de la clínica. Estoy segura que cuando se habla de mujer y psicoanálisis todos pensamos en conceptos como género o transgénero, por eso llegué a pensar que a lo mejor debería haber titulado esta conferencia "El psicoanálisis de género/transgénero"; o "El psicoanálisis transcultural", sin embargo, he elegido el título de hoy porque quiero que hagamos el ejercicio de pensar en personas, en la sociedad en general, aunque partiendo de que cada persona es única (sea hombre, mujer, transexual, etcétera) e intentaré contestar a la pregunta de si hay dilemas propios de ellas en psicoanálisis.

Para entender a las personas, primero hay que tener una mirada hacia lo político - social, porque no podríamos entender la psicología sin la filosofía 5 , la sociología, o la antropología, sin el contexto social en el que vivimos. No se puede permanecer indiferente a ese contexto ${ }^{6}$, 
lo podemos aceptar o rechazar, ya que el sujeto se adapta a su ambiente incorporando de forma activa los mandatos de género en términos de roles femeninos y masculinos.

La sociedad en la que vivimos nos constituye a nivel individual, a nivel de experiencia del self, tanto si nos percatamos de ello como si no lo hacemos; por lo tanto, ni como terapeutas nos podemos quedar al margen, ya que como dice Orange ${ }^{7}$, dialogar con cada paciente, y coconstruir juntos nuevos significados supone un ejercicio de reflexión que tenga en cuenta también las propias confusiones, pre-conceptos y pre-juicios, lo que irá influyendo en la forma de preguntarse e indagar sobre la experiencia subjetiva de vida de cada cual.

No hablo desde el pesimismo, pero vivimos tiempos complejos, en un mundo globalizado, aunque soy consciente de que yo vivo en Europa, y como cuentan los periodistas Bosh y Suanzes en la Revista $5 \mathrm{~W}^{8}$ ya decía el presidente Obama que Europa es más que un mercado, los europeos debemos ser conscientes de que vivimos en el mejor mundo posible: hay democracia, libertad, un alto nivel de educación, menos pobreza, etcétera, por lo tanto, deberíamos ser optimistas, no complacientes. No obstante, se ha ido instalando en el imaginario colectivo cierta sensación de desconcierto ante una crisis económica a nivel global de la que no acabamos de salir, una crisis identitaria que tiene que ver con las migraciones, y un miedo al diferente que propicia cada vez más racismo ya sea explicito o implícito, se está instalando un discurso público tendente al fatalismo. Ya desde la entrada en la postmodernidad asistimos a una ruptura de las estructuras de familia y trabajo tal como las hemos conocido hasta ahora.

Pensemos en el movimiento $M$ e $\mathrm{ToO}^{9}$, en las manifestaciones feministas, tan necesarias para transformar la sociedad machista que hasta el día de hoy ${ }^{10} y$, por desgracia, acarrea la dramática cifra de 42 mujeres asesinadas en España ${ }^{11}$ a manos de sus parejas o exparejas. Hay más información, más movilizaciones, y más sensibilidad social pero, los movimientos en contra adquieren cada vez más fuerza, intentando minimizar la importancia de las consecuencias para la mujer maltratada como son la pérdida de identidad, la situación de vulnerabilidad de la madre y de los hijos, o el largo y espinoso camino de la justicia; reapareciendo también el estereotipo que creíamos ya superado, de que solo las mujeres con dependencia emocional, y bajos recursos económicos pueden ser víctimas de la sociedad patriarcal.

Pensemos, también en las religiones, con sus mandatos y mensajes al grupo social, aunque en el mundo occidental vivimos en una separación de Estado y Religión, nunca es una separación total. Las creencias religiosas, la mitología y los prejuicios están formando parte del imaginario colectivo y contribuyen a la construcción de la identidad colectiva e individual. 
Y no olvidemos que estamos en una sociedad digitalizada, sin referentes claros, donde todo tiene que ser resuelto al instante, hay que tomar decisiones rápidas, nos angustiamos ante el silencio y el vacío (pensemos en el móvil por ejemplo) en un estado de saturación permanente, donde no hay espacio para la calma. Las redes sociales han traído muchas novedades y comodidades, pero también han contribuido a lo que Ortega y Gasset ${ }^{12}$ se refería al hablar de la masa que abraza transversalmente a hombres y mujeres de distintas clases sociales, igualándolos en un ser colectivo y abdicando de su individualidad soberana para adquirir la de la colectividad, para llegar a ser nada más que una parte de la tribu. Resulta difícil diferenciarse del resto de "la tribu", aunque paradójicamente haya una creciente individualidad, como afirma Foucault ${ }^{13}$ cada sociedad produce su propia verdad y como escribe Fromm, la sociedad no es algo abstracto y el individuo no es otra cosa que un ser relacional, somos lo que hacemos y hacemos lo que somos ${ }^{14}$.

Ni nosotros como terapeutas ni los pacientes que atendemos podemos sustraernos a estos datos, a estas informaciones, nos toquen más o menos de cerca, dependiendo del momento vital en que nos encontremos. Lo social entra en las sesiones y actualmente también ha entrado la política, unas veces de forma directa y otras de forma sutil, no hablando de política directamente sino de las emociones que conllevan discursos, manifestaciones, hechos, noticias, actos, sean de humillación, idealización, de valentía o cobardía, en definitiva, actos de comunión con el grupo social.

Antes de seguir, propongo un ejercicio: algunos de los que asistimos en el año 2018 a las jornadas del Instituto de Psicoterapia Relacional ${ }^{15}$, participamos en un taller impartido por nuestro colega inglés Andrew Samuels ${ }^{16}$ que a mí me resultó muy esclarecedor. Hagamos solo uno de los ejercicios que se practicaron en ese taller: que cada uno piense en su primer recuerdo político, ese que nada tiene que ver con un acontecimiento público de relevancia, sino más bien con un sentimiento político sobre algo que le impactó en el entorno familiar.

Y con este ejercicio podemos darnos cuenta de la importancia e interrelación que tiene tanto el discurso familiar como el político-social. Como psicoanalistas estamos comprometidos con la actualidad, y con las problemáticas de la subjetividad y la intersubjetividad. Y esto implica la necesidad de una mirada a: la realidad personal; al carácter individual - el inconsciente individual; el carácter social - el inconsciente social; la raza; la clase social; las creencias religiosas; los traumas, y el pasado que conecta con el presente; el momento presente y la visualización del futuro. Trabajamos con el lenguaje, pero sabemos que el paciente no nos trae solo un lenguaje informativo, sino uno afectivo, lleno de creencias, y de lo sabido no pensado. 
Como terapeutas no podemos sustraernos de nuestra propia subjetividad, aunque la opinión personal no es lo que importa sino nuestra posición clínica, nuestra mirada psicoanalítica, ¿qué somos capaces de ver? o ¿qué no somos capaces de entender? Me pregunto si los psicoanalistas tenemos dificultades para entender otras situaciones que no sean las conocidas por la teoría psicoanalítica clásica; por ejemplo, pensemos en la situación "padremadre-bebé" ¿somos capaces de ir más allá de la interpretación psicoanalítica ortodoxa? ¿qué es lo estándar? ¿en qué teoría nos basamos?

Antes de seguir, me gustaría confirmar que estoy de acuerdo con la mayoría de los autores relacionales, acerca de que el sufrimiento, y el dolor psíquico tienen como base la falta de regulación, y de reconocimiento, y que tiene que ver con la excitación causada por respuestas inadecuadas o abrumadoras y la ausencia de mentalización (ver Mitchell, Bio Bleichmar, Benjamin). No me baso en la teoría de la pulsión y la libido de Freud, ya que el enfoque freudiano se basa en la diferenciación de categorías masculino/femenino; activo/pasivo y en la complementariedad de hombre/mujer, convirtiendo y legitimando la diferencia biológica en desigualdad jerárquica, en relaciones de poder, donde el hombre es libre y la mujer dependiente y necesitada (ver Freud).

Hay un contexto e imaginario psicoanalítico, somos herederos del psicoanálisis ortodoxo, y a mi me gustaría que pensemos la trayectoria del Complejo de Edipo ${ }^{17}$ a lo largo de los años, y nos preguntemos donde está la niña, y la mujer, y qué lugar les dejamos a ellas en ese imaginario.

Si partimos desde finales del siglo XIX hasta los años 50, y sobre todo, los 60 y 70 del pasado siglo XX, vemos que la interpretación clásica del Complejo de Edipo nos traslada a la ley del padre, al paradigma del conflicto entre generaciones, entre lo viejo y lo nuevo, un complejo que representa la lucha por adaptarse a una realidad concreta aunque sea a costa de renunciar a los sueños, y ahí la sexualidad de la niña y de la mujer para Freud ${ }^{18}$ es algo complejo, alguien a quien él no acaba de entender, que está en segundo plano, defendiendo que la complementariedad de la niña con el padre y la no identificación con la madre es lo que constituye la feminidad. Nos encontramos con un deseo neurótico dominado fundamentalmente por la ambivalencia y la culpabilidad.

A partir de los años 60-70 aparece un movimiento anti complejo de Edipo, ${ }^{19}$ que ya se inició en los años 50, y que critica al psicoanálisis por estar al servicio del orden establecido. Aparece un movimiento social que trata de negar toda dependencia de la autoridad de los padres (padre y/o madre) intentando escaparse y prescindir de la ley paterna. Todo esto coincide con la aparición de las primeras mujeres psicoanalistas junto con la llamada $2^{\mathrm{a}}$ y $3^{\mathrm{a}}$ Ola del feminismo ${ }^{20}$. Se empieza a estudiar el deseo de las mujeres dotándolas de voz propia 
y a plantear el deseo sexual como algo separado del deseo del varón, la mujer quiere sentir que es dueña de su propio cuerpo. Nos encontramos con un deseo menos neurótico, con menos culpa y más tendente a lo esquizoide.

A finales de los años 9o, empezamos a comprobar que el narcisismo hace aparición como una tendencia general en la familia y en las políticas de educación, hay una falta de diferenciación entre generaciones, tanto el niño como la niña son el centro de la familia, a la vez que los padres dejan de ejercer cualquier actividad educativa, y tratan de evitar que sus hijos tengan que enfrentarse a la realidad, quedando encerrados en una especie de burbuja especular; es interesante comprobar que cuando se promete solo felicidad y placery se niega la realidad, la vida cotidiana se convierte en una dura prueba a superar constantemente, o incluso una prueba imposible de superar. No hay deseo, más bien frustración y por tanto decepción.

En esta época los movimientos feministas siguen luchando por la igualdad en el terreno público pero en el privado, el narcisismo hace mella de diferente manera en mujeres y hombres. Tomo prestado de Carlos Rodríguez Sutil ${ }^{21}$ los términos narcisismo de piel dura y narcisismo de piel fina, considerando, como él afirma, el narcisismo como algo saludable, como la afirmación de la propia imagen y como la protección frente a las amenazas. Visto así, el narcisismo de piel dura correspondería a los hombres y estaría enfocado a proteger su identidad de varón, dándoles seguridad y asertividad tanto para la vida pública como privada, mientras que el narcisismo de piel fina sería propio de las mujeres y se reflejaría en una afirmación de la imagen pública envuelta en un halo de autoexigencia que a su vez esconde una imagen privada tendente a la vergüenza, la inseguridad y la dependencia.

Actualmente, el psicoanálisis también es criticado, recibiendo ataques como en los años 6o70, pero en este caso, tachándolo de anticientífico, o seudocientífico, se defiende a ultranza la psicología científica, el poder de los fármacos y la autoridad exclusiva de la psiquiatría clásica. ${ }^{22} \mathrm{y}$, paralelamente a estos movimientos anti-psicoanálisis, aparece un movimiento Ilamado por Recalcati ${ }^{23} \mathrm{El}$ complejo de Telémaco. ${ }^{24}$ Atiendo en consulta a jóvenes que están pidiendo, casi exigiendo, como Telémaco, que alguien les haga de padres/madres, pero de padres/madres simbólicos, que tomen las riendas para no sentirse huérfanos, no están en conflicto con los padres/madres, simplemente tienen la esperanza de poder contar con ellos.

En el psicoanálisis la ausencia del padre por sí mismo no tiene porqué ser un trauma, solo lo es si es una ausencia simbólica. La palabra de la madre tiene el poder de significar la ausencia del padre de maneras muy diferentes; estoy pensando, por ejemplo, en las parejas separadas o divorciadas que tienen que hablar por separado cada uno de los progenitores a sus hijos enviándoles mensajes sobre la imagen del otro progenitor, o las familias monoparentales, 
las adopciones, o las parejas homosexuales donde hay dos madres y dos padres. Telémaco no desea un mundo ideal ni otras leyes, sino recuperar esa figura como algo simbólico, no como alguien autoritario. Nos encontramos con un deseo plagado de angustia, de no saber qué va a ocurrir, en el fondo no se sabe lo que se desea, pero me presunto si lo que se desea es tener deseo.

Y también me pregunto qué papel jugaría la madre y la mujer en este deseo de encontrar un orden simbólico, una madre que es capaz de poner también orden y una mujer que tiene deseos propios, y no sé si esto está ocurriendo, más bien en la consulta las pacientes me transmiten que se ven obligadas a reivindicar su papel de autoridad simbólica y de persona con deseos propios, se sienten desprotegidas, pues los mitos y los estereotipos sobre roles masculinos y femeninos en la pareja y la sexualidad planean como nubarrones en la vida cotidiana y en la vida íntima.

En general, ¿qué traen las mujeres a consulta? Según el I Estudio Epidemiológico de FEAP, el EPIFEAP $2018,{ }^{25}$ que se realizó para conocer el perfil de las personas que reciben psicoterapia psicoanalítica en el ámbito nacional, las mujeres manifiestan como primer motivo de consulta: sensación de malestar e insatisfacción; cansancio y tristeza prolongada; dificultad en las relaciones personales; miedos y fobias. Y el motivo secundario de consulta, una vez que se sienten en confianza en el proceso psicoterapéutico son: sensación de malestar e insatisfacción; dificultad en las relaciones personales; cansancio o tristeza prolongada; y conflictos de pareja.

Los motivos aparentes de consulta suelen ser una realidad, pero conllevan ese saber sobre uno mismo/una misma que no se ha podido pensar todavía. Por eso la psicoterapia necesita un tiempo, para que paciente y psicoterapeuta puedan ir pensando lo que está ahí pero no ha sido dicho, no ha podido ser compartido y por lo tanto no puede ser elaborado.

Lo sabido no pensando tiene relación con: la fantasía y el deseo; el self genuino, el verdadero self; los cuidados, la autorregulación, y el reconocimiento; la sexualidad y la agresión; las identificaciones y contraidentificaciones.

La fantasía es el primer representante de lo sabido no pensado; ${ }^{26}$ tiene que ver con el self genuino, el self verdadero de Winnicott y también con la manera en que se ha interiorizado el imaginario, los deseos y la lógica de la madre y de los primeros cuidados, y también con la sexualidad y la agresión que están mezclados con el discurso social y con las identificaciones y contraidentificaciones con la madre y las figuras de apego. Podemos afirmar que lo sabido no pensado contribuye en gran medida a la construcción del género, en términos masculino y femenino. 
Cada mujer es única, y tiene unas circunstancias únicas, pero a todas les une una serie dilemas relacionados con el cuerpo, los cuidados, la sexualidad, la intimidad, la maternidad, ${ }^{27}$ y el trabajo.

Coloco el trabajo en último lugar porque pertenece al ámbito público y la sociedad está luchando para que ese espacio sea un lugar común e igualitario para hombres y mujeres, y en esta lucha debemos seguir, lo que realmente pertenece a lo que nos ocupa en esta conferencia esta relacionado con el espacio intimo. No obstante, los dilemas en el campo laboral no se pueden descartar y pueden ser motivo de otra conferencia, ya que entre otros aspectos a destacar podemos afirmar, a grandes rasgos, que ellas expresan una mayor autoexigencia en el rendimiento académico y laboral, que puede ser debido a diversas y múltiples causas y tiene consecuencias sobre su estado de salud y bienestar.

Entre las mujeres hay cierta obsesión por el cuerpo ya desde la adolescencia, habitando cuerpos sexualizados de niña-mujer, así como una mayor autoexigencia en la búsqueda de experiencias sexuales tempranas y satisfactorias, y una mayor autoexigencia para sí mismas en la pareja, la maternidad, y la familia.

Es en el ámbito de la sexualidad y de las relaciones íntimas y de pareja donde suele surgir ese malestar y sensación de cansancio generalizado. Como expresa una paciente mía de treinta y dos años: Yo puedo hacer cosas, me siento segura en mi trabajo, con mis amigos, en mis relaciones sociales pero en cuestiones de relaciones sexuales y de pareja mis emociones todavía pertenecen a mi familia, ¿cómo ser yo misma y no sentirme sometida a los deseos familiares?.

$Y$ es ahí donde también emergen afectos considerados socialmente negativos, que responden a estereotipos y falsas creencias como son: el egoísmo, la culpa, y la vergüenza.

No es raro oír a una paciente decir que se siente egoísta por hacer lo que ella quiere y no atender las necesidades y deseos de los de su entorno familiar, ya sean padres, pareja o hijos. La mujer suele percibir lo que ella desea como algo no tan valioso como lo que quieren los demás que ella desee. El propio deseo sigue siendo un problema, no lo identifican, está muy impregnado de la ética de los cuidados, saben cuidar pero no cuidarse, porque en el contexto patriarcal el cuidado responde a una ética fundamentalmente femenina que todavía se asocia en exclusiva con las mujeres y está dirigida hacia el cuidado de los familiares.

Este malestar relacionado con la intimidad y que conlleva desregulaciones emocionales suele localizarse en el cuerpo, a través de la somatización y la tristeza generalizada. Es un malestar, cansancio, y dolor en apariencia físicos que les hace preguntarse en primer lugar 
¿estoy fallando yo? ¿en qué estoy fallando? ¿qué es lo que me falla en realidad? ¿cómo estoy en el mundo? ¿por qué me aíslo? y ¿de qué me protejo?

La culpa en psicoanálisis tiene relación con el sentimiento de ceder, de renunciar al propio deseo, de ceder a la responsabilidad que en el fondo una sabe que tiene que tener y por motivos varios no llega a poder hacerse cargo. Tanto Kristeva, Benjamin, como Dimen defienden el sujeto activo, lo que denominan Agency, la capacidad de acción, la que permite no aceptar las presiones para actuar de acuerdo a las normas establecidas, una fuerza necesaria para oponerse a la dominación. Hacerse responsable de lo que una da y de lo que se priva nos pone en relación directa con el enfoque de género y es lo que se aborda en consulta, ya que cada mujer habla desde una problemática propia y distinta aunque el dilema sea común a la mayoría.

El género es una experiencia íntima que pone en marcha toda la gama de las emociones del grupo social de pertenencia, del grupo familiar ${ }^{28}$. El género es anterior al sexo y a la sexualidad ${ }^{29}$, tiene una estructura relacional y se crea en un juego de identificaciones en los que participa la madre como sujeto y también la forma en que ésta se relaciona con su pareja (con el padre biológico y/u otra pareja) y la forma en que el padre se relaciona con la madre (con la madre biológica y/u otra pareja).

En el terreno de la intimidad, aparece el sentimiento de vergüenza como una parte importante en la construcción de la identidad de género y la identidad sexual y también como un potente desregulador de los estados del self.

Se suele manifestar este sentimiento de vergüenza en la intimidad con una misma y con la pareja, e implica a la identidad, es algo que nos es dado y algo que nosotros construimos en un mundo común de experiencias, donde se puede experimentar el "me siento igual que tu y a la vez puedo ser diferente, también hay algo de mi que no puedo apresar ni expresar porque nadie es totalmente transparente para sí..$^{\circ}$ La vigilancia de este sentimiento está en nuestro interior y en el exterior, en nuestro alrededor y se activa tanto con los rechazos sociales como las humillaciones en las relaciones afectivas; por ejemplo, una humillación activa las mismas rutas neuronales que el dolor físico, por eso decimos me duele esta humillación. ${ }^{31}$

La vergüenza se activa en el juego de la seducción y en el de las relaciones sexuales. La seducción tal como está entendida en nuestra sociedad es un juego de poder entre hombre y mujer que se desarrolla en el terreno de lo enigmático, y que sigue un esquema particular: se suele asumir que el hombre es el que inicia este juego, y la mujer es la que pone límites, se 
resiste, y al final sucumbe a los deseos del varón, la mujer suele fingir para estar a la altura de lo que se espera de ella, viviendo ese juego entre avergonzada y temerosa.

Freud expresaba la seducción en cuanto a experiencia traumática de indefensión frente a la sobrestimulación del otro, pero estoy de acuerdo con Benjamin ${ }^{32}$ en que esta experiencia del exceso puede llevar a la escisión entre la parte activa de una misma persona y su parte pasiva, es decir a la escisión entre lo considerado masculino y lo femenino 33 en un mismo individuo. Actualmente vemos en consulta lo que se refleja en nuestro entorno: el dilema sobre "el consentimiento", "el No es No", "el qué pensará de mí", porque se sigue identificando seducción con abusos, y no se detecta muy bien donde está el límite. Parece que tenemos que reescribir estos esquemas de la seducción, y me pregunto, como hace la socióloga Olivia Muñoz-Rojas ${ }^{34}$ ¿nos quedamos con las tesis del Me Too3, norteamericano, radical y tendente al puritanismo, o con el Me Too francés, que defiende la libertad del "No es No" con matices?. El mundo anglosajón y el mundo francés. Lo dejo como reflexión.

En definitiva y para terminar, podemos afirmar que sí hay dilemas propios y comunes a todas las mujeres en psicoanálisis. Cada persona (mujer, hombre, transexual, queer, etcétera) necesita y quiere ser oída y sostenida y este deseo se expresa mediante significantes de género, como un problema de identificaciones femeninas y masculinas en una misma persona. El problema de género es central en psicoterapia, ya que los significantes de género los tendremos que abordar, sostener y elaborar como personas y como psicoterapeutas junto a nuestros pacientes.

\section{REFERENCIAS}

Ávila Espada, A. (ed). (2013). La tradición interpersonal, perspectiva social y cultural en psicoanálisis. Perspectiva social y cultural en Psicoanálisis. Col. Pensamiento Relacional. Vol. № 8.

Bauman, Z. (2009). En busca de la política. Fondo de cultura económica. Original en inglés de 1999.

Benjamin, J. (2012). Descifrando el enigma del sexo: Pasividad femenina y dominancia masculina, una solución al problema del exceso. Revista CEIR. Vol. 6 (2) - Junio 2012; pp. 187-203

Bollas, C. (1997). La sombra del objeto. Psicoanálisis de lo sabido no pensado. Amorrortu editores

Bosh, A; Suanzes, P.R; (2019). Europa soy yo. Voces. Revista 5W. No 4.

Castaño, R. (2011). La terapia sexual. Una mirad relacional. Editado por Ágora. Col. Pensamiento Relacional. Vol. No 4 . Madrid.

Dimen, M (2003). Sexuality, intimacy, power. The Analytic Press. 
Dimen, M. (2015). Too much Goodness: Another Hazard on the Road to Sexual Boundary: Commentary on Dianne Elise's "Unraveling Betrayal an the Loss of Goodeness in the Analitic Realtionship"Psychoanalytic Dialogues, 25: 572-578. Routledge. Taylor and Francis Group.

Dio Bleichmar, E. (2002). Sexualidad y género. Nuevas perspectivas en el psicoanálisis contemporáneo. Revista Perspectivas psicoanalíticas. No 011

Esquirol, J. M (2015). Uno mismo y los otros. De las experiencias existenciales a la interculturalidad. Herder

Esquirol, J. M. (2015). La resistencia intima. Ensayo de una filosofía de la proximidad. Acantilado.

Foucault, M. (2019). Microfísica del poder. Siglo XXI editores. (Selección de textos de la obra Dits et écrits 1954-1988)

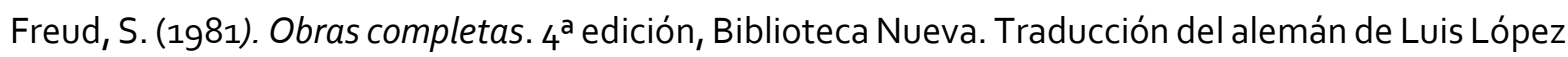
Ballesteros y de Torres

Freud, S. (1924). La disolución del Complejo de Edipo Tomo III pp 2748

Freud, S. (1905). Tres ensayos sobre una teoría sexual. Tomo II pp 1169

Freud, S. (1931). Sobre la sexualidad femenina Tomo III pp. 3077

Freud, S. (1931). La feminidad. Lección XXXIII Tomo III, pp. 3164

Fromm, E. (2011). Espíritu y sociedad. Barcelona, Paidós, Biblioteca Nueva Erich Fromm, original de 1996. p. 74 .

Fromm, E (2012). El lenguaje olvidado. La introducción a la comprensión de los sueños, los mitos y cuentos de hadas. Paidós Biblioteca Nueva Erich Fromm. Original en inglés de 1951

Fromm-Reichman, F. (1989). Principios de psicoterapia intensiva. Hormé Psicología de Hoy. Original de 1950

Harris, A. E. (2011). Gender a a Strange Attractor: Discussion of the transgender Synposium. Psychoanalytic Dialogues, 21:230-238. Routledge.Taylor and Francis Group.

Harris, A. E. (2009). Gender as Soft Assembly. Routledge.

Horney, K. (2010). La personalidad neurótica de nuestro tiempo. Paidós Estudio. Original de 1981

Lipovetsky, G. (2008). La sociedad de la decepción. Anagrama. Original en francés de 2006

Orange, O; Atwood, G.E.; Stolorow R.D. (2012). Trabajando intersubjetivamente, contextualismo en la práctica psiconalítica. Col. Pensamiento Relacional. Vol. № 6

Ortega y Gasset, J. (2019). La rebelión de las masas. Editorial Planeta. De la primera edición en este formato 2009; Herederos de José Ortega y Gasset, 1929, 2005.

Peteiro, J. (2010). El autoritarismo científico, Miguel Gómez Ediciones.

Recalcati, M. (2014). El complejo de Telémaco. Padres e hijos tras el ocaso del progenitor. Anagrama.

Rodríguez Sutil, C. (1998). El cuerpo y la mente. Una antropología wittgensteiniana. Col. Quipú, Biblioteca Nueva. 
Rodríguez Sutil, C. (2014). Psicopatología psicoanalítica relacional. La persona en relación y sus problemas. Col. Pensamiento Relacional. vol. 12.

Sennett, R. (2006). La corrosión del carácter. Las consecuencias personales del trabajo en el nuevo capitalismo. Anagrama. Original en inglés de 1998.

Serrano, V. (2011). La herida de Spinoza. Felicidad y política en la vida posmoderna. Anagrama.

Thompson, C. (1995). El Psicoanálisis. Fondo de cultura económica. Original en inglés de 1950.

Winnicott, D.W. (2002). Los procesos de maduración y el ambiente facilitador. Estudios para una teoría del desarrollo emocional. Paidós. Psicología Profunda. Original en inglés de 1965.

Original recibido con fecha: 15/12/2019 Revisado: 30/12/2019 Aceptado: 15/03/2020

NOTAS:

${ }^{1}$ Para más información consultar en feap.es y en psicoterapiaspsicoanaliticasfeap.info

${ }^{2}$ Centro Médico Instituto Palacios de Salud de la mujer. www.institutopalacios.com

${ }^{3}$ Fromm, E (2012). El lenguaje olvidado. Paidós. Original de 1951

4 Dilema: según la Real Academia Española. (RAE) una situación en la que es necesario elegir entre dos opciones igualmente buenas o malas. Filosóficamente hablando, es un problema que puede resolverse a través de dos soluciones pero ninguna resulta plenamente aceptada, implicando siempre cuestiones morales, éticas y/o sentimentales.

${ }^{5}$ Rodríguez Sutil, C. (1998). El cuerpo y la mente. Una antropología wittgensteiniana. Col. Quipú, Biblioteca Nueva.

${ }^{6}$ Afirmación que suele ser aceptada por la mayoría de los psicoanalistas actuales y por todo el pensamiento relacional y que viene avalada por estudios como los del Grupo de Boston.

7 Orange, O; Atwood, G.E.; Stolorow R.D. ( 2012) Trabajando intersubjetivamente, contextualismo en la práctica psiconalítica. Col. Pensamiento Relacional. Vol. No 6

${ }^{8}$ Bosh, A; Suanzes, P.R. (2019) Europa soy yo. Voces Revista 5W. No 4.

9 Me Too, en español "Yo también," es el nombre de un movimiento iniciado de forma viral como hastag en las redes sociales. Surgió en octubre de 2017 para denunciar abusos sexuales, agresiones y violaciones contra el productor de cine y ejecutivo estadounidense Harvey Weinstein; y desde entonces este hastag ha sido utilizado por más de 500 . 000 personas, entre ellas muchas celebridades.

104 de octubre de 2019.

${ }^{11}$ Según el CIS de 2019, los españoles/as sitúan la preocupación por la violencia machista entre los tres primeros problemas del país. El 47.3\% de las victimas están en una edad entre los 25 y 39 años y las que están en la edad entre 15 y 44 años son las que tienen más riesgo.

${ }^{12}$ Ortega y Gasset, (2019) La rebelión de las masas. Ed. Planeta.

13 Foucault, M. (2019). Microfísica del poder. Siglo XXI editores.

${ }_{14}$ Ávila Espada, A. (ed). (2013). La tradición interpersonal, perspectiva social y cultural en psicoanálisis. Perspectiva social y cultural en Psicoanálisis. Col. Pensamiento Relacional. Vol. No 8

15 V Jornadas de Psicoterapia Relacional (2018) Pareja y Comunidad. Transformando nuestras relaciones cotidianas. Jornadas organizadas por el Instituto Psicoterapia Relacional. Taller impartido por Andrew Samuel. La Granja, Segovia.

${ }^{16}$ Samuels, A, es psicoterapeuta, profesor, activista y escritor. Profesor de Psicología Analítica en Centre for Psychoanalytic Studies, University of Essex (U.K.). Práctica privada como consultor y analista Jungiano.

${ }^{17}$ El complejo de Edipo es un concepto psicoanalítico ortodoxo que Freud crea apoyándose en el mito griego de Edipo, hijo de Layo y Yocasta, abandonado por su padre y adoptado por el rey de Corintio y su esposa. Esta

(c) Derechos reservados/Copyright de Clínica e investigación Relacional y los autores. Prohibida la reproducción total o parcial sin autorización expresa. Este material es para uso científico y profesional exclusivamente y puede contener información clínica sensible. Los editores no se responsabilizan de los contenidos de los autores. Dirigir las consultas sobre derechos y autorizaciones a ceir@psicoterapiarelacional.es 
teoría sostiene que las relaciones familiares del niño/niña están motivadas por fantasías de deseo sexual hacia el progenitor del sexo contrario, el asesinato del progenitor rival y miedo a la venganza mediante la castración en el varón, o inhibición debido a la envidia de pene en la niña.

${ }^{18}$ Ver los escritos de Freud sobre la sexualidad de la niña y sobre la feminidad.

${ }^{19}$ Movimiento anti-Edipo que empieza tímidamente en los años 50 representado por psicoanalistas como Frieda Fromm-Reichman; los culturalistas Harry S. Sullivan; Karen Horney; Clara Thompson, también Erich Fromm; Harold Searles. (ver Ávila, 2013).

${ }^{20}$ Las psicoanalistas Adrianne Rich; Nancy Chodorow; Ethel Parson; Jessica Benjamin; Judith Butler; Virginia Goldner; Muriel Dimen; Dio Bleichmar; y las voces de la $2^{\mathrm{a}}$ y $3^{\mathrm{a}}$ Ola del feminismo Simon de Beavoir; Kate Millet; o Betty Friedan.

${ }^{21}$ Rodríguez Sutil, C (2014) Psicopatología psicoanalítica relacional. La persona en relación y sus problemas. Col. Pensamiento Relacional. vol. 12

22 Peteiro, J. (2010) El autoritarismo científico, Miguel Gómez Ediciones.

${ }^{23}$ Recalcati, M. (2014) El complejo de Telémaco. Padres e hijos tras el ocaso del progenitor. Anagrama. Ver también Gilles Lipovetsky, Richard Sennett, Vicente Serrano, Zygman Bauman.

${ }^{24}$ El complejo de Telémaco: En la Odisea de Homero, Telémaco, el hijo de Ulises es abandonado por su padre que se ve obligado a ir a la guerra de Troya, tardando 20 años en volver, mientras tanto el hijo sufre en su casa el acoso y los desmanes de príncipes y de pretendientes que quieren casarse con su madre Penélope. El hijo se dedica a buscar a su padre para que venga a poner orden, pide ayuda a los mayores, y espera con deseo su regreso.

25 EPIFEAP (2018). Promovido por la Junta Directiva de la Sección de Psicoterapias Psicoanalíticas de FEAP: Rosario Castaño; Miguel Perlado; Beatriz C. Bonanata; Javier Naranjo; Pablo Juan J. Maestre; coordinado por Ricardo Gallego, y con el apoyo de FEAP bajo la presidencia de Iñigo Ochoa de Alda Martínez de Apellaniz, y aprobado por el Comité Ético del Hospital de la Princesa de Madrid.

${ }^{26}$ Bollas, C. (1997). La sombra del objeto. Psicoanálisis de lo sabido no pensado. Amorrortu editores

${ }^{27}$ Según el Instituto Nacional de Estadística (INE) un 4\% de los padres de niños menores de 8 años opta por la reducción de jornada frente al $26 \%$ de las mujeres en la misma situación. Se penaliza la maternidad de forma indirecta si no hay disponibilidad total para la empresa. Ver también La política del flexiworking y El club de las malas madres.

${ }^{28}$ Castaño, R. (2011). La terapia sexual. Una mirada relacional. Editado por Ágora. Col. Pensamiento Relacional. Vol. No 4. Madrid

${ }^{29}$ Dio Bleichmar, E. (2002). Sexualidad y género. Nuevas perspectivas en el psicoanálisis contemporáneo. Revista Perspectivas psicoanalíticas. No 011

$3^{\circ}$ Esquirol, J. M (2015) Uno mismo y los otros. De las experiencias existenciales a la interculturalidad. Herder.

${ }^{31}$ Ver autores como Dimen, Harris; Benjamin; Bio Bleichmar.

${ }^{32}$ Benjamin, J. (2012) Descifrando el enigma del sexo: Pasividad femenina y dominancia masculina, una solución al problema del exceso. Revista CEIR. Vol. 6 (2) - Junio 2012; pp. 187-203

33 Sí podríamos considerar el concepto femenino como sinónimo de pasividad y el masculino, como de actividad, tal como lo expresaba Freud aunque para él lo masculino se instalaba en el varón y lo femenino en la mujer, pero para el psicoanálisis relacional, lo femenino y masculino se dan en una misma persona, independientemente de que sea un cuerpo de varón, mujer o trans.

34 Olivia Muñoz-Rojas ${ }^{34}$ Los dilemas de la seducción, El País del 29 de septiembre de 2019. 